\title{
A SYMMETRIC REPRESENTATION OF THE TWENTY- SEVEN LINES ON A CUBIC SURFACE BY LINES IN A FINITE GEOMETRY*
}

\section{J. S. FRAME}

1. Introduction. The group $G_{51840}$ of the automorphisms of the twenty-seven lines and forty-five tritangent planes on a general cubic surface has an even subgroup $G_{25920}$ which is simple. This may be represented on the one hand as the linear group $A(4,3)$, and on the other hand as the linear group $H O(4,4) . \dagger$ Each of these linear groups suggests a representation of the configuration of the lines on the cubic surface by lines in a finite geometry. Coble $\$$ has analysed the invariant configurations of the finite projective geometry $P G(3,3) \S$ under the group $A(4,3)$. In this paper we shall examine those configurations of the $P G(3,4)$ under the group $H O(4,4)$ which are isomorphic to the configurations of lines and planes on the general cubic surface.

The notation to be developed in this paper assigns coordinates in a symmetric manner to the twenty-seven lines and forty-five tritangent planes on the cubic surface and affords extremely simple conditions to determine their incidences. For this reason it has some advantages over the commonly used double-six notation devised by Schläfli.\|

2. The planes. We assign to the planes of the $P G(3,4)$ a set of four homogeneous coordinates $\left(u_{0}, u_{1}, u_{2}, u_{3}\right)$ which are marks of the field $F \equiv G F\left(2^{2}\right)$. The four marks of $F$, which we denote by $0,1, \omega, \bar{\omega}$, are roots of the congruence

$$
u^{4} \equiv u(\bmod 2)
$$

* Presented to the Society, February 26, 1938.

$\dagger$ L. E. Dickson, Linear Groups, 1901, p. 309. J. S. Frame, The simple group of order 25920, Duke Mathematical Journal, vol. 2 (1936), p. 477.

$¥$ A. B. Coble, A Configuration in Finite Geometry Isomorphic with That of the Twenty-seven Lines of a Cubic Surface, Johns Hopkins University Circulars, no. 208, 1908, pp. 80-88.

$\S \mathrm{R}$. D. Carmichael discusses finite projective geometries in his Introduction to the Theory of Groups of Finite Order, 1937.

\| L. Schläfli, On the twenty-seven lines upon a surface of third order, Quarterly Journal of Mathematics, vol. 2 (1858), pp. 110-120. A. Henderson, The Twenty-seven Lines upon the Cubic Surface, 1911. For other notations, see H. S. M. Coxeter, Polytopes with regular-prismatic vertex figures, Philosophical Transactions of the Royal Society of London, vol. 229 (1930), pp. 396, 418. 
Defining the conjugate $\bar{u}$ of a mark $u$ to be its square, we use the abbreviation

$$
\sum_{i=0}^{3} \bar{u}_{i} v_{i}=u \cdot v,
$$

and we call two planes, $\left(u_{0}, u_{1}, u_{2}, u_{3}\right)$ and $\left(v_{0}, v_{1}, v_{2}, v_{3}\right)$, orthogonal if $u \cdot v=0$.

The group $H O(4,4)$ leaves invariant the bilinear form $\boldsymbol{u} \cdot \boldsymbol{v}$. Of the $\left(4^{4}-1\right) /(4-1)=85$ planes $\boldsymbol{u}$, the forty-five for which $\boldsymbol{u} \cdot \boldsymbol{u}=0$ are permuted transitively among themselves, and so are the remaining forty for which $u \cdot u=1$. We shall call the former isotropic, and the latter non-isotropic. Upon inspection we find that each of the fortyfive isotropic planes is orthogonal to twelve others, forming three sets of four linearly dependent planes. For example, the plane $\boldsymbol{u}:(1,1,1,1)$ is orthogonal to each of the twelve planes $k u+v$, where $k \varepsilon F$, and $v$ is one of the three planes $(0,0,1,1),(0,1,0,1),(0,1,1,0)$. If $\mathbf{u}$ and $\mathbf{v}$ are any two mutually orthogonal isotropic planes, just five isotropic planes $k u+l_{v}$ pass through their line of intersection. Each plane contains just three such lines, which form a triangle. Each line meets ten others, which lie by pairs in the five incident isotropic planes. This proves the isomorphism described in the following theorem:

THEOREM 1. The forty-five tritangent planes to the general cubic surface are in one-to-one correspondence with the forty-five isotropic planes of the finite geometry $P G(3,4)$, in such manner that two tritangent planes which intersect in a line of the surface correspond to two isotropic planes which are orthogonal.

3. The lines. A simple notation for the line of intersection of two isotropic planes $u$ and $v$ may be derived from the six Pluecker coordinates, $p_{i j}=u_{i} v_{j}-u_{j} v_{i}$, of the line. These coordinates are connected by the identity

$$
p_{01} p_{23}+p_{02} p_{31}+p_{03} p_{12}=0 .
$$

The conditions $\boldsymbol{u} \cdot \boldsymbol{u}=\boldsymbol{v} \cdot \boldsymbol{v}=\boldsymbol{u} \cdot \boldsymbol{v}=0$ can be shown to imply that both of the factors in one of the terms of equation (3) must vanish. The other four determinants $p_{i j}$ must be different from zero if the planes $\boldsymbol{u}$ and $\boldsymbol{v}$ are distinct. Two suitably chosen ratios of these four quantities, together with the relation (3), suffice to determine a line. In order to preserve symmetry, while avoiding division by zero, we may use the conjugate of a mark of the field $F$ instead of its reciprocal. Accordingly, we assign to a line the three non-homogeneous coordinates

$$
\left(\bar{p}_{01} p_{23}, \bar{p}_{02} p_{31}, \bar{p}_{03} p_{12}\right),
$$


of which just one is zero. For each of the three positions of the zero, there are nine ways of choosing the other two coordinates. These twenty-seven possibilities correspond to the twenty-seven lines on the cubic surface.

In terms of these coordinates, the condition that two lines of the cubic surface intersect is simply that they have just one coordinate in common.

THEOREM 2. The twenty-seven lines of the general cubic surface are in one-to-one correspondence with the twenty-seven number triples

$$
(0, b, c),(a, 0, c),(a, b, 0), \quad a, b, c=1, \omega \text {, or } \bar{\omega},
$$

in such manner that two intersecting lines correspond to two triples having just one coordinate in common. The three sides of a triangle on the surface correspond to two such triples and their vector sum (mod 2).

To prove this theorem, we verify that the line $(0, b, c)$ is the line of intersection of the planes $(b c, 1,0,0)$ and $(0,0, b, c)$. The three planes $(a b c, a, b, c)$, linearly dependent on these two, form with them the set of five planes which contain the given line $(0, b, c)$. In each plane there are two other lines:

Plane

$$
\begin{aligned}
& (b c, 1,0,0) \\
& (0,0, b, c) \\
& (b c, 1, b, c) \\
& (\omega b c, \omega, b, c) \\
& (\bar{\omega} b c, \bar{\omega}, b, c)
\end{aligned}
$$

\section{Lines}

$$
\begin{aligned}
& (0, \omega b, \bar{\omega} c),(0, \bar{\omega} b, \omega c) \\
& (0, \omega b, \omega c),(0, \bar{\omega} b, \bar{\omega} c) \\
& (1,0, c), \quad(1, b, 0) \\
& (\omega, 0, c), \quad(\omega, b, 0) \\
& (\bar{\omega}, 0, c), \quad(\bar{\omega}, b, 0) .
\end{aligned}
$$

These ten lines are characterized by the simple property that they each have just one coordinate in common with the line $(0, b, c)$. Furthermore, $(0, b, c)$ is the vector sum $(\bmod 2)$ of the coordinates of each of the five pairs of these lines which intersect the line $(0, b, c)$ and form with it a triangle. Similar arguments for the lines $(a, 0, c)$ and $(a, b, 0)$ complete the proof of Theorem 2 .

4. The trihedral pairs. If we now fix our attention on the forty nonisotropic planes of the $P G(3,4)$, we find that each is orthogonal to twelve others. In this set of twelve planes, each is orthogonal to two others, which in turn are mutually orthogonal. Hence there are $40 \times 12 \times 2 / 4 !=40$ sets such as$$
(1,0,0,0) \text {, }
$$$$
(0,1,0,0) \text {, }
$$$$
(0,0,1,0)
$$$$
(0,0,0,1) \text {, }
$$ 
consisting of four mutually orthogonal non-isotropic planes of the $P G(3,4)$.

THEOREM 3. The forty triads of trihedral pairs of tritangent planes to the general cubic surface are isomorphic with the forty sets of four mutually orthogonal non-isotropic planes of the $P G(3,4)$.

Proof. Each such set of planes $W, X, Y, Z$ in $P G(3,4)$ may be divided in three ways into two pairs, such as $W, X$ and $Y, Z$. The pair $W, X$ determines three mutually non-orthogonal isotropic planes $W+k X,(k=1, \omega, \bar{\omega})$, which correspond to a triheder of three tritangent planes to the surface which do not intersect each other on the surface. The two associated pairs, $W, X$ and $Y, Z$, correspond to a pair of conjugate triheders enjoying the property that each plane of the first cuts each plane of the second in a line of the surface. Since the two planes $W+k X$ and $W+l Y$ are not mutually orthogonal, the three trihedral pairs formed from the set $W, X, Y, Z$ have no common lines of the surface but exhaust the twenty-seven lines in three sets of nine lines.

5. The group. The group of unitary transformations of the $P G(3,4)$, of determinant unity, transforms the forty triads of trihedral pairs among themselves. Furthermore, the four columns of the matrix of each transformation are a mutually orthogonal set of homogeneous coordinates for the transforms of the four non-isotropic planes (7). Altogether there are 4 ! permutations of the four columns, and $3^{3}$ ways of multiplying the columns by factors without altering the value of the determinant. This proves that the order of the group is $40 \times 3^{3} \times 4$ ! $=25920$. A transformation of period two not contained in this group changes each coordinate to its conjugate mark in $F$. The direct product is a group of order 51840, which is isomorphic with the group of automorphisms of the twenty-seven lines on the general cubic surface.

Brown UNIVERSITY 